Irene Dölling

\title{
Frauen- und Männerbilder
}

\author{
Eine Analyse von Fotos in DDR-Zeitschriften
}

In den letzten Jahren ist in der DDR bei Philosoph/innen und Gesellschaftswissenschaftler/innen ein zunehmendes Interesse an der Lebensweise, an Formen des Alltagslebens und des Alltagsbewußtseins feststellbar. Hintergrund dieses Interesses sind praktische Erfahrungen bei der Gestaltung der sozialistischen Gesellschaft: sozialokkonomische Verhältnisse und individueller LebensprozeB sind vielfach vermittelt über Formen, die oft eine längere, mehrere historische Gesellschaften übergreifende Geschichte haben; gesamtgesellschaftliche, Gruppen- und individuelle Interessen sind keineswegs homogen, sondern von großer Differenziertheit, die sich in vielfältigen Formen Ausdruck verschafft; die Art und Weise, wie sich Welt-Anschauung unmittelbar, im praktischen Lebensvollzug der Individuen bildet, weist Eigentümlichkeiten auf usw.. In der Kulturwissenschaft hat dies z.B. zu einer stärkeren Beschäftigung mit kulturell - symbolischen Formen, mit kulturellen Wahmehmungs-, Denk- und Wertungsmustern geführt, mittels derer Individuen ihren alltäglichen Lebensproze $B$ regelhaft gestalten, ihren Handlungen einen Sinn geben. $\mathrm{Zu}$ diesen Mustern gehören u.a. die stereotypen Vorstellungen von "Weiblichkeit « und "Männlichkeit«, die in einer Kultur »gang und găbe « sind. Sie sind wie eine Art Raster. durch das »hindurch« reale Gegebenheiten wahrgenommen, geordnet, gewertet werden: z.B. die verănderte Situation von Frauen durch Gleichberechtigung, lebenslange Berufstătigkeit und sozialpolitische Maßnahmen bei gleichzeitig fortwirkender Verantwortung für die biologische Reproduktion und für die Hausarbeit, sowie unter den Bedingungen einer ausgeprägten Arbeitsteilung zwischen den Geschlechtern in der gesellschaftlichen Produktion. Diese Muster sind im Alltag der Individuen auf vielfache Weise existent und wirksam - so beiläufig wie selbstverständlich eine bestimmte Sicht auf die Welt konstituierend und praktisches Handeln orientierend. Nicht zuletzt werden sie im Zeitalter der Massenmedien bildhaft, in sinnlichanschaulicher Gestalt (und in endloser Wiederholung) verbreitet ${ }^{1}$.

Im folgenden sollen einige Ergebnisse einer umfangreicheren Untersuchung zu Frauen - und Männerbildem auf Fotos in DDR-Zeitschriften mit Massenauflage ${ }^{2}$ vorgestellt werden ${ }^{3}$. Die Fragen, die die Analyse der Zeitschriftenfotos leiteten, waren: 
- Wie werden auf Bildem von Frauen und Mănnem die Verănderungen, die sich durch proklamierte Gleichberechtigung, lebenslange Berufsarbeit von Frauen usw. vollziehen, in der alltäglichen Realităt ihrer Beziehungen sichtbar - anschaulich und Anschauung bildend?

- Welche Aspekte der Geschlechterverhältnisse werden auf den Fotos abgebildet, welche sind nicht bildwürdig (und warum nicht)?

- Wie werden auf den Fotos aus dem DDR-Alltag normsetzende Vorstellungen über das Verhältnis von Frau und Mann, von »Weiblichkeit« und »Männlichkeit« an-schaulich vermittelt und damit eine bestimmte Sicht auf die Widersprüchlichkeit praktizierter Geschlechterverhältnisse als selbstverständlich nahegelegt?

- Haben die bisherigen Veränderungen in der Lebenssituation von Frauen und Männem zu einem Aufbrechen tradienter Geschlechterstereotype geführt, sind neue entstanden oder ist ein weitgehend ungebrochenes Wirken tradierter Vorstellungen von »Weiblichkeit« und »Mănnlichkeit« zu konstatieren?

Um ein Ergebnis der Analyse vorweg zu nehmen: Es konnten keine neuen Stereotype aufgefunden werden, die ein qualitativ anderes Verhalmis von Frauen und Männem zueinander in eine »feste Form gießen«. Mit wenigen Modifizierungen sind es die überkommenen, patriarchalisch geprägten Vorstellungen von »Weiblichkeit« und »Mănnlichkeit«, mittels derer die reale Situation von Frauen und Männern sinnlich - anschaulich in eine Ordnung gebracht wird. Dies soll im folgenden an einigen wenigen Beispielen gezeigt werden. Sie wurden aus dem Bereich gewăhlt, in dem sich bzw. durch den sich tatsächlich die größten Veränderungen in der Lebenssituation von Frauen (bzw. in den Geschlechterverhältmissen) vollzogen haben: der Berufsarbeit. Die $Z$ wischenüberschriften verdeutlichen, daß dabei der Schwerpunkt auf die Frauenbilder gelegt wird.

Das „schöne, schwache Geschlecht" ist leistungsfähig und steht in der gesellschaftlichen Produktion "seinen Mann"

Berufstätigkeit von Frauen ist in der DDR eine Selbstverständlichkeit geworden. Über $90 \%$ aller Frauen im arbeitsfahigen Alter üben gegenwärtig einen Beruf aus bzw. befinden sich in der Ausbildung. Fotos, die Frauen bei der Arbeit zeigen, machen dementsprechend in den analysierten Zeitschriften den gröBten Anteil aus (sie werden nur in der FD noch übertroffen von Abbildungen weiblicher Mannequins). Auf sehr vielen Fotos aus den 70er und 80er Jahren repräsentieren berufstätige Frauen weniger das »schöne« oder »schwache« Geschlecht, sondern die auf sachliche Arbeitsergebnisse, auf Leistung orientierte Produzentin. Beim Betrachten dieser Fotos springt etwas unmittelbar ins Auge: Zwar ist in der Regel auf den ersten Blick zu erkennen, daß die abgebildeten Personen Frauen sind. $\mathrm{Zu}$ gleich aber drängt sich durch eine Abschwächung bestimmter Stereotype von "Weiblichkeit" die Geschlechtszugehörigkeit nicht in den Vordergrund der Wahrnehmung. Das trifft vor allem auf Fotos zu, die Frauen in ihre Abeit vertieft zei- 
gen $^{4}$. Die sinnlich - anschauliche Vermittlung găngiger Stereotype von $*$ Weiblichkeit« spielt auf ihnen kaum eine Rolle: wie die abgebildeten Frauen gekleidet und frisiert sind, ob ihre Figur den $»$ Idealmaßen entspricht, ob sie geschminkt sind und vorteilhaft« ins Bild kommen - dies alles tritt zurück hinter die Konzentration, mit der die Frauen bei der Sache sind, hinter die Fertigkeit, mit der sie mit Werkzeugen oder Maschinen umgehen, hinter die fachliche Kompetenz, die an ihren Mienen und Gesten ablesbar ist. Leistung ist gefragt (auch für die abgebildeten Frauen selbst), und die Fotos vermitteln den Betrachtem genau dies - in Einheit mit den Unterschriften - als Eindruck.

Es ist eine Besonderheit der kulturellen Formen, daB sie in ihrer bildhaften "Sprache « vieldeutig sind. Was also kơnnte es bedeuten, daß auf den charakterisierten Fotos aus dem Berufsleben die Frauen weniger als das mschönek oder »schwache $\ll$, sondern dominant als das leistungsfahige Geschlecht erscheinen? Welche Widerspriichlichkeiten der aktuellen Situation von Frauen bzw. der Geschlechterverhältnisse könnten damit durch unterschiedliche Bedeutungen anschaulich gemacht werden? Eine Bedeutungsebene, die so ins Bild gesetzt wird, ist, daB Frauen in der gesellschaftlichen Produktion unentbehrliche Arbeitskrafte sind. Wie die Mănner leisten Frauen gesellschaftlich notwendige und anerkannte Arbeit, oftmals mit dem Einsatz von Fähigkeiten und Fertigkeiten, die nach traditionellem Muster zur »männlichen « Skala gehðren. Indem auf den Fotos diese geschlechtstypischen Zuweisungen durch den selbstverständlichen Habitus der Frauen durchbrochen werden, können bestimmte Eigenschaften und Făhigkeiten als menschliche (und nicht geschlechterspezifische) und können Frauen - im Kontext der Berufsarbeit - weniger als Geschlechtswesen und mehr in ihren tatsächlich entwickelten, "weibliche« wie »männliche« Anteile gleichermaßen umfassenden subjektiven Qualitäten wahrgenommen werden. Ich vermute allerdings, daß diese Bedeutungsebene gegenwărtig, unter den Bedingungen einer ausgeprägten geschlechterspezifischen Arbeitsteilung in der Produktion, (noch) eine untergeordnete Rolle spielt. Sie deutet mehr eine mogliche Perspektive an und symbolisiert weniger alttäglich gewordene Realităt. Sinnlich - anschaulich zeigt sich dies auch daran, daß auf vergleichbaren Fotos von Männem bei der Berufsarbeit eine ăhnliche Kombination »mănnlicher « und »weiblicher« Făhigkeiten nicht feststellbar ist. Fotos von Männem bei der Arbeit »sprechen« vielmehr auf anschauliche Weise davon, daß sich für die Vertueter des »starken« Geschlechts in der, wesentlich um die Berufsarbeit zentrierten, Bestimmung ihrer Geschlechter-»rolle « nicht vicl geändert hat. Auch bilden Berufsarbeit, Be-herrschung von Maschinen und Prozessen durch Kopf- oder Handarbeit eine so unauflösliche Einheit, daß es keiner Abschwächung von »männlichen« Stereotypen bedarf, um Männer als leistungsfähig zu zeigen. Das ist anders als bei Frauenbildem, weil die Stereotype der »Weiblichkeit « traditionell auf andere "Bestimmungen « des weiblichen Geschlechts verweisen. Auf den Fotos scheinen z.B. durch die gewählte Perspektive Technik und Männer(körper) miteinander zu verschmelzen: raumgreifende Gesten, die kraftvollen Körper, die mit ihren Bewegungen die Technik kontrollieren, aufmerksamer Blick und lockere, entspannte Haltung, die Kompetenz und das Identisch-sein mit der Aufgabe ausdrücken - all dies signalisiert, daß Männer in dieser Welt »zu Hause« sind. Frauen dagegen 
werden oft so fotografiert, daß sie klein gegenüber mächtigen technischen Apparaten erscheinen, die den Bildraum beherrschen.

Bisher hat sich die Einbeziehung der Frauen in die Berufsarbeit in Form einer ausgeprägten geschlechterspezifischen Arbeitsteilung vollzogen. Unter diesen Bedingungen hat die Abschwächung von Weiblichkeitsstereotypen noch andere (und gegenwărtig dominierende) Bedeutungen, die die »Verarbeitung « einer widersprüchlichen Realităt in der individuellen Wahmehmung und Wertung orientieren. Sie signalisiert, z.B., daß die Bereiche bezahiter Berufsarbeit zwischen den Geschlechtem aufgeteilt sind, die herkömmliche »Ordnung «, nach der Männer in den entscheidenden Tatigkeiten und Positionen »das Sagen« haben, nicht in Frage gestellt ist. Sinnlich - anschaulich wird das etwa dadurch, da B weitaus häufiger Frauen oder Männer, als beide Geschlechter gemeinsam bei der Arbeit abgebildet sind. Zum anderen sprechen « die Fotos, die zu einer Reportage oder einem Artikel gehören und die verschiedenformatig z.B. den Leiter, die mit der Einrichtung neuester Technik beschaftigten Mănner sowie am FlieBband arbeitende Frauen zeigen, mittels Reihung von dieser Realität. Sie erscheint auf diese Weise als so selbstverständlich, wie zugleich wenig bedrohlich für den Bestand einer hierarchischen Geschlechteranordnung. Auf den Fotos muß daher auch nicht durch Betonung der Schönheit und sinnlichen Verführungskraft des weiblichen Geschlechts eine reale Gefahr verkleinert, von ihr durch Verschiebung abgelenkt werden. Zugleich werden andere Aspekte des »weiblichen Geschlechtscharakters« auf den Fotos durchaus betont. Die große Anzahl von Fotos von Frauen in »typischen « Frauenberufen wie Kindergärtnerin, Lehrerin, Krankenschwester, bzw. in den unmittelbaren Fertigungsbereichen der industriellen Produktion geben nicht nur die Tatsache geschlechterspezifischer Arbeitsteilung wieder. Mit der Hăufigkeit der Abbildungen von Frauen in diesen Berufen werden auch besonders anschaulich die stereotypen Muster von der dienenden, unterstützenden, helfenden, fürsorgenden Funktion und "Bestimmung " des weiblichen Geschlechts positiv bestätigt und reproduziert. Damit wird aber auch das Neue, also die selbstverständliche, kompetente und verantwortliche Berufsarbeit von Frauen,als etwas ins Bild gesetzt, was die găngigen Geschlechter-»rollen « nicht grundsätzlich in Frage stellt. Die Abschwächung von Weiblichkeitsstereotypen auf den Fotos hat in diesem Kontext eine spezifische Bedeutung. Dies hängt aufs engste zusammen mit der bildsprachlichen Möglichkeit des Identisch-machens von Ungleichartigem. Indem auf diesen Fotos Frauen vordergnündig als leistungsfähige Produzentinnen und erst in zweiter Linie als »weibliche « Wesen erscheinen, werden die formale Gleichstellung sowie die Gemeinsamkeiten mit den berufstätigen Männem (bezahlte, fachlich kompetente Arbeit in der gesellschaftlichen Produktion) in die Aufmerksamkeit der Wahrnehmung gerückt. Damit wird einerseits eine wichtige Veränderung in den Geschlechterverhältnissen und in der gesellschaftlichen Anerkennung der Frau veranschaulicht und zugleich wird mit diesem Identisch-machen die Tatsache der geschlechterspezifischen Arbeitsteilung in der Berufssphäre in den Hintergrund der Wahmehmung gerückt. Dadurch wird der Fakt verkleinert, daß Frauen auch in der »neu eroberten« Sphäre praktisch benachteiligt sind und auf dem altbekannten "zweiten Rang « belassen werden ${ }^{5}$. 


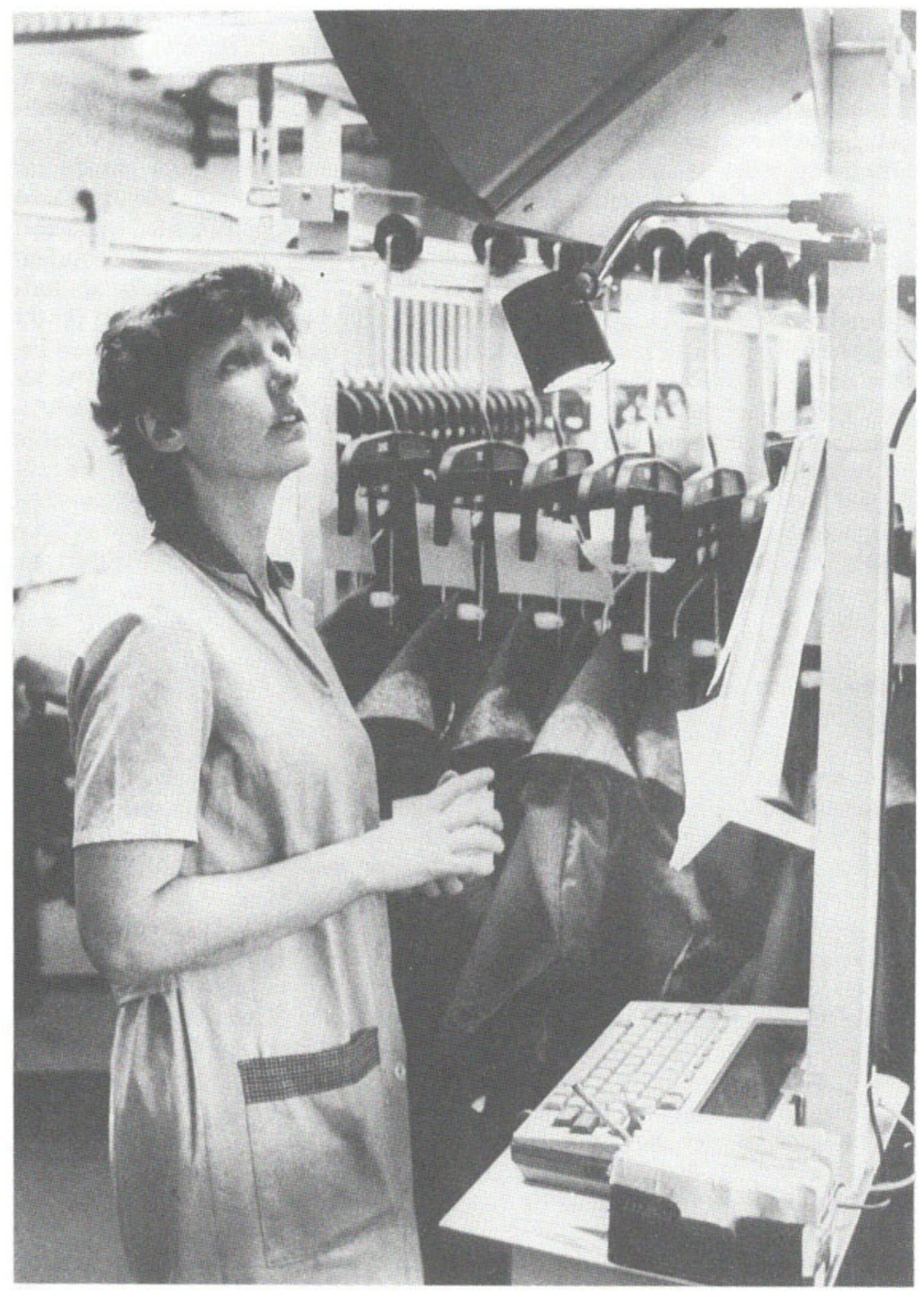

Dateneingabeplatz für das automatisierte Transportsystem in der Sakkoendfertigung. Petra Büttner « füttert « den Computer mit Informationen. Dann erfolgt kontinuierlich die Belieferung der 40 Arbeitsplätze mit Sakkoteilen (Quelle: »NBI*, 2187, S. 9, Fotograf: Heinz Dargelis) 
Bis heute ist Berufstătigkeit eine $*$ mămliche火 Angelegenheit in dem Sinne, daB die Kriterien für Effizienz, Leistungsfahigkeit, berufliches Engagement usw. vom »Idealtypus« der »männlichen* Arbeitskraft bestimmt sind, vom mănnlichen Erwachsenen also, dessen Leben inhaltlich und zeitlich wesentlich von Berufsarbeit geprägt ist und der eine (weibliche) »Hintergrundsperson« hat, die für seine Reproduktion und seine Kinder sorgt. Gemessen an diesen Kriterien sind berufstătige Frauen, unabhăngig von ihrer beruflichen Qualifikation, »anders «; sie können ihnen immer nur teilweise genügen. In ihrem Leben sind die Aufgaben bei der biologischen Reproduktion und die - bislang fast ungebrochen fortwirkende - Verantwortung für den Haushalt mindestens genauso wichtig für ihr Selbstverstăndnis »als Frauk. (Laut soziologischen Untersuchungen rangieren Familie und Ehe bei Frauen aller Altersgruppen vor der Berufsarbeit in der Werteskala ${ }^{6}$ ). Fotos nun, die ihre traditionell verstandene $\star$ Weiblichkeit« in der Berufssphäre »unterbelichten«, dürften daher auch dieses Selbstverstăndnis bekräftigen und bestătigen. Die Fotos geben so zwar Berufsarbeit als selbstverstăndliche, alltägliche Erfahrung von Frauen wieder, aber auch als etwas das ihr „Frau-sein nicht oder noch nicht wesentlich tangiert. Und damit wird indirekt auch auf ihre meigentliche Bestimmung* verwiesen, weshalb im veroffentlichten Leitbild die „Frau von heute« nicht die Berufstätige, sondern die berufstatige Mutter ist ${ }^{7}$.

\section{Wenn Frauen das Gleiche tun wie Männer, ist es nicht unbedingt dasselbe!}

Wenn auch nicht so hăufig wie Fotos von Individuen, die einzeln oder in gleichgeschlechtlichen Gruppen ihrer Arbeit nachgehen, finden sich in den Zeitschriften selbstverstăndlich auch Fotos, die Frauen und Mănner gemeinsam bei der Arbeit zeigen. Selten sind dabei allerdings Fotos, auf denen Frauen und Männer bei gleichen Tätigkeiten zu sehen sind - an den gleichen Maschinen oder mit den gleichen Gerăten arbeitend, in synchronen Bewegungen und Haltungen, die Aufmerksamkeit und die Kraft auf dasselbe Ziel gerichtet. Weitaus häufiger sind Fotos, auf denen mittels bestimmter Geschlechterstereotype die Beziehungen der gemeinsam arbeitenden Frauen und Mănner so strukturiert werden, daß anschaulich wird: es ist nicht dasselbe, wenn Frauen und Männer das Gleiche tun. Ein Bildtyp zieht sich dabei über die Jahrzehnte (bei wechselnden konkreten Zusammenhängen) durch die Zeitschriften: Mann belehrt, unterweist Frau. Es gibt Fotos, die in der Beziehung der abgebildeten Personen zueinander eine geschlechterspezifische Arbeitsteilung, ein Unterstellungsverhältnis, eine soziale Hierarchie unmittelbar, direkt, sinnfällig machen (Chef unterweist und kontrolliert Sekretărin). Auf vielen Fotos aber geht es um Arbeitszusammenhănge, in denen Frauen und Männer die gleichen Tätigkeiten ausüben. Auch diese Abbildungen sind von einer Bild-»sprache " geprägt, die den Mann als den kompetenteren zeigt, als denjenigen, der Bescheid weiß, der den Überblick und das Sagen hat: er wird durch die gewählte Perspektive optisch vergrößert, seine Überlegenheit wird sichtbar in seiner ruhigen Körperhaltung, dem sachkundigen Blick, der sicheren, erklärenden Geste, während sie fragend zu ihm aufblickt, oft in angespannter 


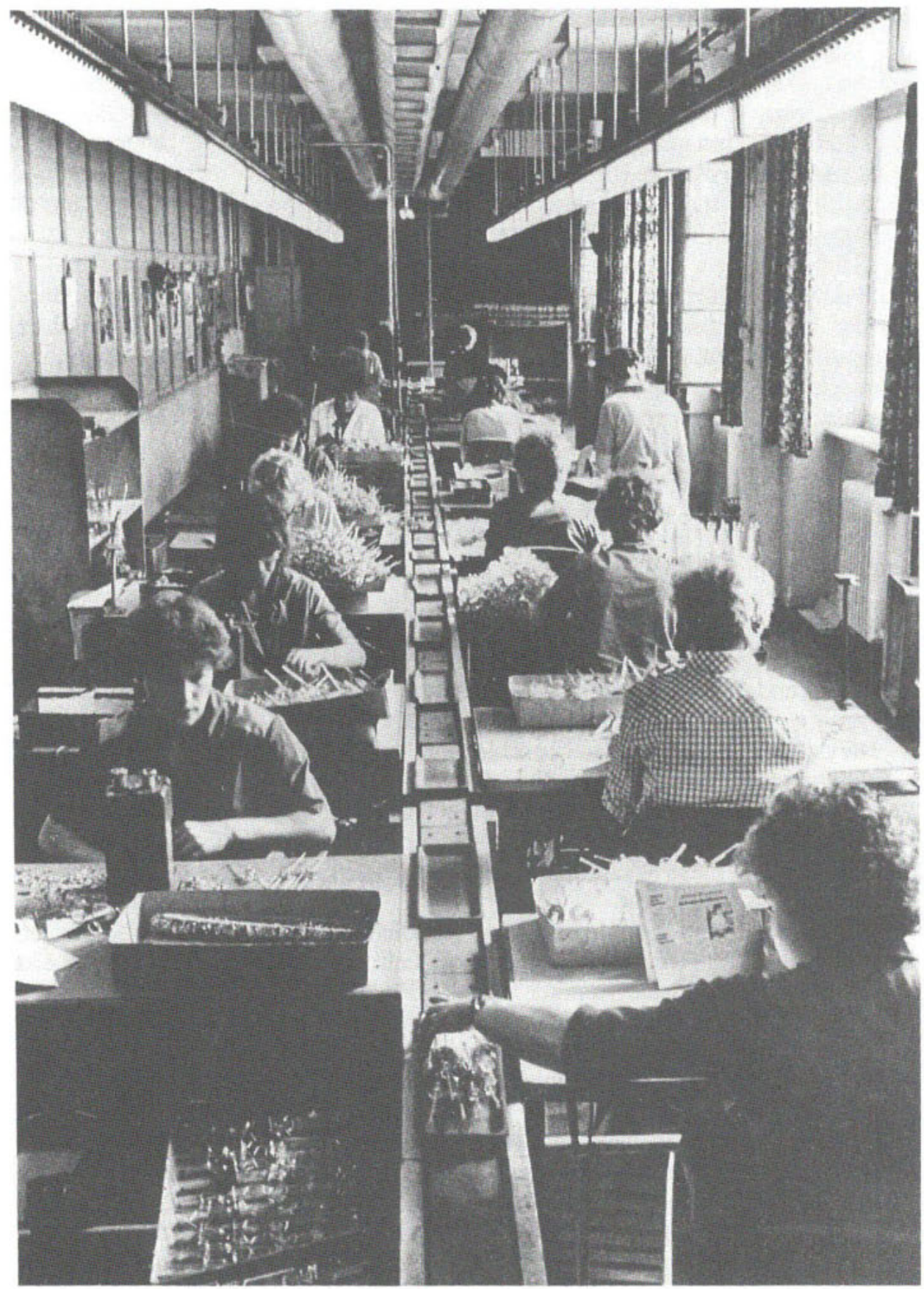

Glas braucht behutsame Hände ... Die Technologie bestimmt hier die Sitzordnung, der zugekehrte Rücken hat nichts mit dem Verhältnis der Frauen untereinander zu tun (Quelle: ॠ ür Dich*, 4/88, S. 14, Fotograf: Andreas Kämper) 
Körperhaltung den Erläuterungen lauscht oder unter den begutachtenden Augen des Mannes eifrig bemüht ist, nach den gegebenen Hinweisen die Arbeit gut zu machen. Das Foto von den beiden jungen Frauen vor dem Computer und dem Mann, der im Vordergrund mehr als UmriB und doch die Szene beherrschend ins Bild kommt, ist ein gutes Beispiel dafür. Der Habitus der beiden Frauen, ihr sachkundiger, aufmerksamer Blick drückt Kompetenz und Sicherheit aus, nur wenig haben sie noch mit den Frauen auf den Fotos aus den 60er Jahren gemeinsam, die sich etwas zaghaft und unsicher der ungewohnten Technik năherten. Dennoch ist die Szene in einem Moment festgehalten, wo der Mann in Aktion ist. Verstärkt noch durch die sitzende, bzw. beidhändig aufgestützte Haltung der Frauen, durch die der Mann optisch vergroßert wird, durch den Gegensatz von Aktivität des Mannes (Sprechen, lebhafte Gestik) und Passivität der Frauen (lauschende, aufnehmende Haltung) wird die Kooperation von Mann und Frauen nach tradierten Geschlechterstereotypen veranschaulicht. Den Betrachtern, für die die konkreten Zusammenhänge ohnehin in der Regel unbekannt und kaum von Interesse sind, prägen sich in der Wahrnehmung vor allem die »typischen « Haltungen, Gesten usw. von Frauen bzw. Mănnern im Kontext neuer Zusammenhănge (moderne Technologien) ein, die in ihrer sinnlichen Gestalt vermitteln: "So " sind Frauen und Mănner (und so sollen sie bleiben).

Auch für die Versinnbildlichung sozialer Differenzierungen und Hierarchien werden Geschlechterstereotype auf unterschiedliche Weise eingesetzt. Besonders anschaulich wird dies auf Fotos, die Leiter/innen in ihren Arbeitskollektiven zeigen. Als Tendenz láßt sich feststellen:

- Handelt es sich um gleichgeschlechtliche Gruppen, werden die durchaus sichtbaren Hierarchien (Hand- und Kopfarbeiter/innen, Leiter/innen und Geleitete) optisch vermindert durch eine bildliche Anordnung, die »Gleichrangigkeit«, Gleichwertigkeit hinsichtlich der zu erreichenden Aufgabe symbolisiert und auf diese Weise die auch unter Frauen bzw. Mănnem vorhandenen Hierarchien verkleinert.

- Je geringer die Unterschiede auf der benflichen Stufenleiter zwischen Leitem und weiblichen Arbeitskollektiven bzw. Leiterinnen und männlichen Arbeitskollektiven sind, desto weniger werden Geschlechterstereotype eingesetzt zur Veranschaulichung »männlichen« Führungsanspruchs bzw. des »Rollentausches«. Zwar »sprechen« z.B. die immer wiederkehrenden Fotos von Frauen, die sihren Meister« im Zentrum des Bildes umrahmen, davon, wer der Chef ist. Aber der befindet sich in der Regel optisch kaum vergroßert, ohne größere Distanz, inmitten "seiner " Frauen. Fotos dieser Art sind nach meinem Überblick auch beinahe die einzigen, auf denen spielerisch mit einem Anflug ironischer Brechung, die Realität einer geschlechterspezifischen Arbeitsteilung und das Wissen von Frauen um diese Tatsache ebenso wie um ihre fachlichen Fähigkeiten und ihre Unersetzbarkeit in »Szene gesetzt « werden.

- Jeder Hauch von ironisch-kritischer Distanz fehlt dagegen auf den Fotos von hochqualifizierten Frauen und Männern in höheren Leitungspositionen. Wie auch, stellt doch der »Einbruch « von (relativ wenigen) Frauen in Bereiche mit größerer Verantwortung, Informiertheit, Übersicht und Entscheidungsbefug- 


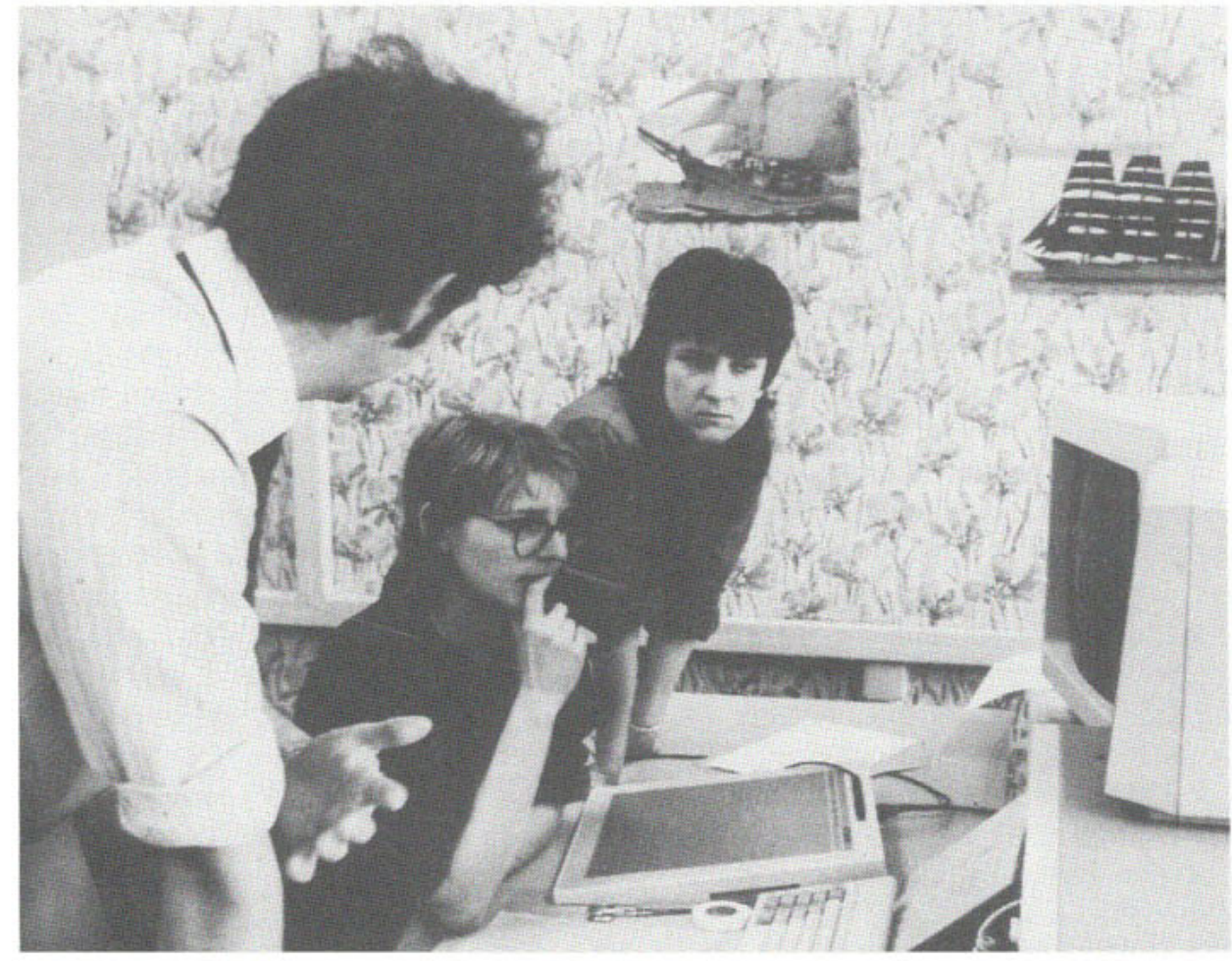

(Quelle: „Für Dich*, 18/88, S. 12/13, Fotograf: Andreas Kamper)

nis, eine hergebrachte hierarchisierte Ordnung (nicht nur in der Produktion) weitaus mehr in Frage, als die Übernahme eines Meisterbereiches. Kein Wunder also, da $B$ auf solchen Fotos hochrangige Leiter, optisch auffallend vergroBert, in großer Distanz zu den Frauen abgebildet werden, die in der beruflichen Qualifikation und Hierarchie weit unter ihnen stehen. Für die wenigen Frauen, die bis zu solchen Hoben gekommen sind ${ }^{3}$, wird eine Bild-»sprache « gewăhlt, die der oben erwăhnten Abschwăchung von Weiblichkeitsstereotypen diametral entgegengesetzt ist und auf die in einem letzten Punkt eingegangen werden soll.

Je qualifizierter verantwortungsvoller, exklusiver die beruflichen Tätigkeiten von Frauen sind, desto mehr wird das Bild vom leistungsfähigen weiblichen Geschlecht durch Hervorhebung von "Schönheit" und "weiblichen" Körperformen weichgezeichnet

Entsprechend ihrem Ziel, die berufliche und politische Gleichberechtigung der Frauen in der DDR zu propagieren, stellt die FD relativ häufig »erfolgreiche" Frauen in herausragenden Tătigkeiten und Funktionen vor ${ }^{9}$. Auffallend ist, daß quasi entgegengesetzt zur intendierten Botschaft realisierter Gleichberechtigung 
- auf den zu den Reportagen und Berichten gehörenden Fotos diese *Vorzeigefrauen mit demonstrativer Betonung tradierter Stereotype von »Weiblichkeit ins Bild gesetzt werden. Das trifft insbesondere auf die meist groBformatigen, farbigen Aufmacherfotos $z \mathrm{u}^{10}$. Ebenso wie die Titelfotos entsprechender Frauen, die es mit »Konsequenz und Charme , mit „Charme und Logik«, "konsequent und verständnisvoll « geschafft haben, in ihrem Beruf erfolgreich zu sein, präsentieren diese Aufmacher die Frauen vordergründig als wweibliche "Wesen: das Haar fällt weich und gepflegt, die schmeichelnde Seidenbluse harmoniert mit der Farbe der strahlenden Augen, die Haut des Gesichts ist so leuchtend und rosig, daB die Fältchen gar nicht auffallen, das Pink der Bluse und des Lippenstiftes stimmen haargenau überein usw.. Fachliche Kompetenz und hochrangige Position werden auf diese Weise verkleinert, (reale oder potentielle) Gefăhrdung einer hergebrachten Ordnung dadurch gemildert, daß diese werfolgreichen* Frauen als verkörperte »Weiblichkeit« erscheinen, deren Verlust so auch als die eigentliche, angstmachende Gefahr signalisiert wird. Zudem ist in solchen bild-»sprachlichen« Arrangements auch eine subtile Form der Abwertung von Frauenarbeit feststellbar. Auf dem Foto der als $»$ Agraringenieurk apostrophierten Frau, die in anmutiger Haltung in einem duftigen Sommerkleid, neben sich die hochhackigen Schuhe, mit bloBen FüBen (wnach alter Bauemerfahrung «) die Lagertemperatur des Getreides in einem Silo prüft, ist die Szene ja nicht nur nach găngigen Stereotypen von "Weiblichkeit" strukturiert. Das ganze Arrangement steht in scharfem Kontrast zu den (»mănnlichen«) Eigenschaften wie: kühle Sachlichkeit, die Zurücknahme individueller Eigenarten, hinter die »Pflichten «, was sich bis in die beherrschte Haltung und die uniforme, unauffallige Kleidung außert, die nach găngigen Vorstellungen von Menschen in gehobenen Funktionen und Tătigkeiten erwartet (und die auch fortlaufend durch Fotos von Männem in hochrangigen Berufen anschaulich bestătigt) werden. Indem die Aufmachung und Präsentation der Frau eher »Freizeit«, »Spaziergang am Sonntagnachmittag « assoziiert als »ernsthafte (»männliche «) Arbeit, schwingt darin auch unterschwellig mit, daB die Arbeit von »Frau Agraringenieur « nicht die Qualität hat (haben kann), wie die von »Herrn Agraringenieur «.

Ich möchte an dieser Stelle die Beschreibung der Geschlechterstereotype, nach denen Bilder aus dem Berufsalltag von Frauen und Mănnem in DDR - Zeitschriften geordnet, strukturiert sind, abbrechen. Sie dürfte hinreichend verdeutlicht haben, wie ungebrochen patriarchalisch geprägte Stereotype von »Weiblichkeit « und »Mănnlichkeit« zur bildhaft-anschaulichen Vermittlung und Wertung einer Realităt dienen, die durch den unaufgelosten Widerspruch zwischen proklamierter Gleichberechtigung und ausgeprägter geschlechterspezifischer Arbeitsteilung in der gesellschaftlichen Produktion gekennzeichnet ist. Vollständig wird das Bild ohnehin erst, wenn auch die Fotos aus den anderen Bereichen des individuellen alltăglichen Lebens einbezogen werden (unbezahlte Arbeit zur individuellen Reproduktion, Versorgungs- und Freizeitgemeinschaft »Familie «, Mutterund Vaterschaft usw.). Erst in Einheit mit den vielen Fotos aus der »Privatsphäre«, auf denen die "Mütterlichkeit« der Frauen als ihr »eigentliches Wesen « herausgestellt wird ${ }^{11}$ und in Berücksichtigung der Tatsache, daB bei den $»$ Szenen aus dem Alltag« fast völlig Fotos fehlen, die Frauen (und Männer) bei der Haus- 


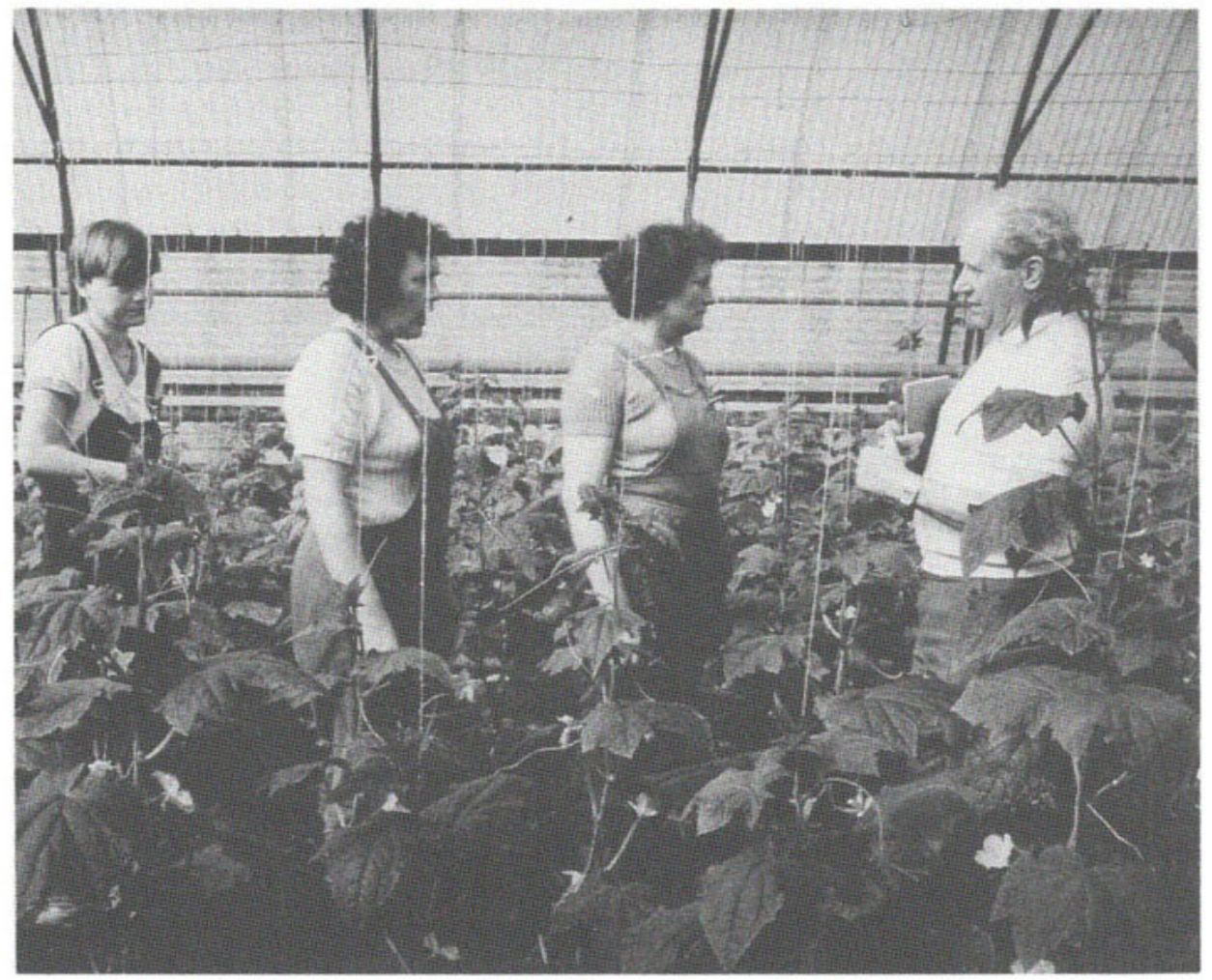

Lange Reihe: Dr. Käßler vom Institut für Gemüseproduktion überprüft jeden Donnerstag mit "seinen Frauen das Forschungsprogramm in den Versuchshallen. Hier werden die Grundlagen der bis 1990 geplanten Produktionssteigerungen gelegt (Quelle: $»$ Für Dich«, 17/86, S. 28/29, Fotograf: Wilfried Glienke)

arbeit $^{12}$ zeigen, wird sichtbar, daB die tradierten Stereotype von $*$ Weiblichkeit火 und "Mănnlichkeit« auf den Fotos aus dem Benufsleben die Wahmehmung nicht nur auf die Akzeptanz einer (zur Zeit nicht sofort aufhebbaren) geschlechterspezifischen Arbeitsteilung in der gesellschafulichen Produktion orientieren. Sie vermitteln (im Kontext aller anderen Fotos) Interpretationsangebote für eine Wirklichkeit, die, ungeachtet des Einstiegs der Frauen in die lebenslange, qualifizierte Berufsarbeit, bislang durch keine qualitativen Änderungen in den geschlechterspezifischen Zuweisungen von Tätigkeiten, Verantwortlichkeiten, Entscheidungsräumen usw. gekennzeichnet ist. Auch hat sich gezeigt, daB die groBzügig ausgebauten sozialpolitischen Maßnahmen, so hilfreich sie im einzelnen sind, in ihren bisherigen Formen tradierte Geschlechterverhältnisse so gut wie nicht in Frage stellen und in dieser Hinsicht in aller Regel eher eine konservierende Wirkung haben. Und als noch allgemeiner und übergreifender erweisen sich solche Interpretationsangebote, wenn gefragt wird nach den politischen Strukturen und den ideologischen Zielen, aus denen sich Vorstellungen einer sozialen Hierachie, von Macht und Unterordnung fortlaufend reproduzieren - unter anderem in ihrer »na- 
türlichsten«, »selbstverstăndlichsten« Form einer Rangordnung der Geschlech$\operatorname{ter}^{13}$.

Die Frage liegt nahe: Welchen Nutzen hat eine solche Fotoanalyse und das Konstatieren einer fast ungebrochenen Existenz tradierter Stereotype von »Weiblichkeit« und "Männlichkeit«? Bevor ich eine (vorläufige) Antwort versuche, möchte ich eine Grenze des praktizierten Vorgehens benennen. Die auf den Fotos erkennbare Virulenz patriarchalisch geprägter Geschlechterstereotype sagt zum einen nicht direkt etwas aus über die tatsächlichen Veränderungen in der sozialen und rechtlichen Stellung der Frauen, über Veränderungen in ihrer beruflichen Qualifikation, ihren individuellen Fähigkeiten, ihrem Selbstverständnis, ihrer Kooperation mit den Mănnem in den verschiedenen Lebensbereichen. Zum anderen können bisher nur Vermutungen darüber angestellt werden, wie die skizzierten Muster, die bildhaft-anschaulich für die Wahmehmung, Deutung und Wertung der widersprüchlichen und konflikthaften Verhälmisse zwischen den Geschlechtern (als Ausdruck gesellschaftlicher Widersprüche) in den Medien angeboten werden, tatsächlich von Frauen und Männern auf- und angenommen werden. Es gibt keine soziologischen Untersuchungen, die sich die Dokumentation dieser individuellen »Verarbeitungen « zum Gegenstand machten. Das schränkt die Aussagefăhigkeit des gewăhlten Verfahrens ein.

Gewonnen werden kann damit ein schărferer und sensiblerer Blick für die unbewußt wirkenden, in der Regel unterhalb des Diskurses bleibenden kulturellen Formen und Muster, die unseren Alltag regeln, durch die reale Veränderungen in einen Wahmehmungs- und Wertungsrahmen gepreBt werden, der bestehende Strukturen nicht in Frage stellt, ihr Infragestellen verhindert. Bislang wurde die wirklichkeitskonstituierende Măchtigkeit solcher kulturellen Muster in der historisch-materialistischen Gesellschaftstheorie stark unterschătzt. Die bis heute verbreiteten Vorstellungen von der Emanzipation der Frauen durch ihre Einbeziehung in die gesellschaftliche Produktion, ergänzt durch eine Sozialpolitik für sie, sind unmittelbarer politischer Ausdruck eines solchen reduktionistischen Verständnisses. Einer kritischen Sicht auf herkömmliche gesellschaftliche Arbeitsteilungen und Wertungen, die die notwendigen Tätigkeiten für die individuelle Reproduktion zur Nicht-Arbeit und zur Frauen-Arbeit machen, auf die Existenz und Wirksamkeit patriarchalischer Strukturen in allen Lebensbereichen, ist ein solches Verständnis nicht förderlich. Über einen solch relativ engen empirischen $\mathrm{Zu}$ gang, wie die Analyse von Zeitschriftenfotos können neben einer Bilanz widersprüchlicher Resultate von bisheriger "Frauenemanzipation « im Sozialismus auch diese angedeuteten Ausblendungen und Verkürzungen in der Theorie wie generell die allgegenwärtige Existenz einer praktischen und symbolischen $\mathrm{Ge}$ schlechteranordnung zur Sprache gebracht, nach ihren Ursachen geforscht werden. Zugleich gewinnen diese Fragen im Kontext aktueller Reform - und Emeuerungsprozesse in den sozialistischen Ländem eine größere, perspektivische Dimension $^{14}$ : Neben einer strategischen Orientierung im Konzept eines modernen Sozialismus bezüglich der Zukunft gegenwärtiger geschlechterspezifischer Arbeitsteilungen, geht es auch und nicht zuletzt darum, das tradierte Denkmuster einer »selbstverständlichen « Geschlechterrangordnung der radikalen Kritik zu unterziehen. Ohne dies ist weder die Artikulation und Akzeptanz spezifischer In- 


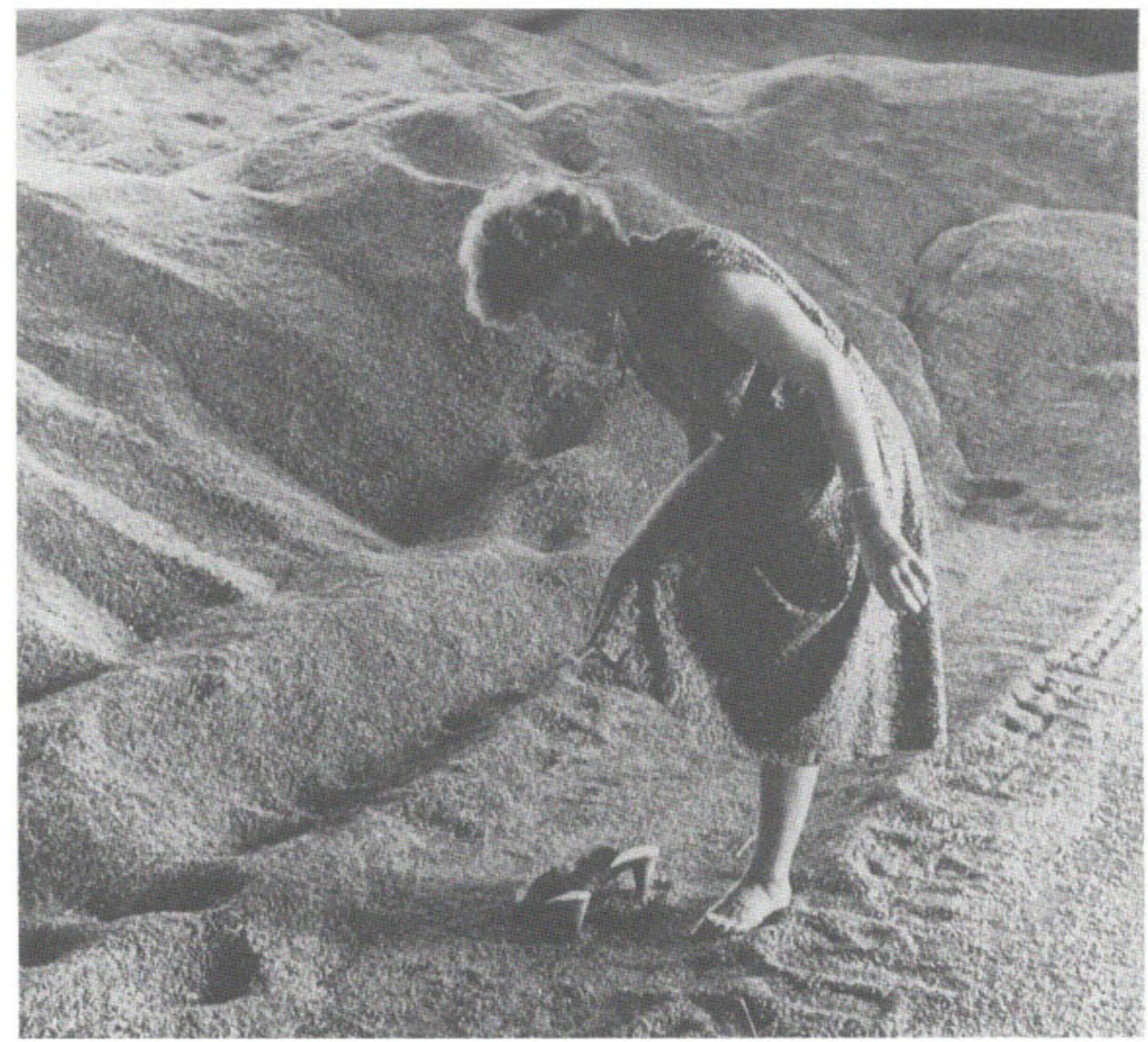

Mit nackten Füßen ins Korn, um die Lagertemperatur des Getreides festzustellen. Besonders in den Ecken der Halle, wo man mit dem Thermometer schlecht hinkommt, wendet Edda van Riesen gern diese alte Bauernerfahrung an. Wird das Korn zu warm, muß es umgeschaufelt werden. Sonst sinkt die Qualität

(Quelle: „Für Dich«, 33/87, S. 14, Fotografin: Katja Worch)

teressen von Frauen (in ihrer Differenziertheit und Verschränktheit mit anderen sozialen Lagen) möglich und erfolgversprechend, noch die Entwicklung von politischen Strukturen, die qualitativ auf die Aufhebung der Teilung in Mächtige, Entscheidungsbefugte und Informierte einerseits, in Untergeordnete, Ausführende, Uninformierte andererseits zielen. Der im globalen Menschheitsinteresse liegende Übergang zu Dialogfähigkeit und Toleranz, zu einem Naturverhältnis, das sich nicht auf den Herrschaftsgedanken gründet, wird nur gelingen, wenn auch im alltäglichen Verhalten die Vorstellung einer "natürlichen « Zweitrangigkeit des weiblichen Geschlechts keinen Platz mehr hat. Ein solches Um- und Neudenken kann nicht in schlichter Entgegensetzung zum »alten Denken « entstehen - es schließt ein, danach zu fragen, welchen Gewinn Frauen und Männer unter gegebenen Bedingungen aus dem Festhalten am Hergebrachten ziehen (können). Die 
Antwort kann nicht von Expertinnen theoretisch abgeleitet, sondem muB vor allem vor den »betroffenen« Subjekten selbst gegeben werden. Dazu ist ein ProzeB offentlicher Verstăndigung notwendig, in dem Erfahrungen und Erkenntnisse miteinander vermittelt werden müssen. GewiB ändert sich durch »neues Denkenk allein nicht die Wirklichkeit. Aber ebenso ist sicher: Erst wenn im Alltag das "Selbstverständliche* patriarchalisch geprägter Stereotype von »Weiblichkeit" und »Männlichkeit « nicht mehr als selbstverständlich angesehen wird, sind in der Realităt Möglichkeiten zu entdecken für Geschlechterverhăltnisse, in denen der »kleine Unterschied« keine soziale Rangfolge bedeutet.

\section{Anmerkungen}

1 In diesem doppelten Sinn wird der Terminus *Frauen- und Mănnerbilder* hier verwendet: es geht um Bilder von Frauen und Münnem (in unserem Falle um Fotos aus dem DDR-Alltag), uber deren sinnlich-konkrete Gestaltung normsetzende Vorstellungen von *Weiblichkeit* und *Männlichkeit « an-schaulich vermittelt werden.

2 Es handelt sich um die jeweils wöchentlich erscheinenden Zeitschriften Newe Berliner Illustrierte (NBI) und Für Dich (FD). Die NBI informien in Wort und Bild uber Weltgeschehen und DDR-Alltag. Sie hat keine spezifische Zielgnuppe, sondem wendet sich sozusagen ^an alle«. Die FD ist die einzige Frauenzeitschrift der DDR. Sie stellt sich die Propagienung der neuen, gleichberechtigten Stellung der Frauen in der Gesellschaft und ihrer Entwicklung in Beruf und Politik zum Ziel. Dadurch ergeben sich Akzentsetzungen in Themen- und Fotoauswahl, die einerseits dazu fuhren, daß DDR-Alltag im Vergleich zur NBI anders, mit anderen Gewichtungen ins Bild kommt. Andererseits - und vielleicht gerade deshalb - tritt in der FD der Widerspruch zwischen propagiertem Leitbild und den Geschlechterstereotypen, nach denen auf den Fotos Wirklichkeit geordnet und gewertet wird, besonders deutlich zutage. Bei der Fotorecherche wurde in mehreren Schritten vorgegangen. Zum einen wurden im Zehnjahresabstand je ein Jahrgang der Zeitschriften durchgesehen und nach einem bestimmten quantitativen Verfahren ausgewertet. Fur die Jahrgănge 1960 bzw. 1986 wurden nach diesem quantitativen Verfahren Feinanalysen gemacht. Zum anderen wurden - ausgehend von charakteristischen Veranderungen bzw. Schwerpunkten jeweiliger Frauenpolitik von den 50er bis zu den 80er Jahren - mehrere Jahrgănge der Zeitschriften durchgesehen, um die Hàufigkeit von (Bild)Themen bzw. "typische " Bildarrangements herauszufinden.

3 Ergebnisse dieses Projektes werden voraussichtlich 1991 in Buchform beim Dietz-Verlag, Berlin unter dem Titel Der Mensch und sein Weib. Aktuelle Frauen- und Männerbilder, ihre geschichtlichen Ursprünge und Perspektiven veröffentlicht.

4 Das sind in der Regel kleinformatige schwarz-weiß Aufnahmen, die oftmals zu Berichten oder Reportagen uber einen Betrieb, uber volkswirtschaftlich wichtige Vorhaben, die Einfuhrung neuer Technik usw. gehören. Dabei werden Frauen in Arbeitsabläufen gezeigt, deren bildliche Wiedergabe im Vordergund steht - oft informieren die Bildunterschriften in erster Linie uber diese und nicht oder nur beiläufig uber die abgebildeten Frauen.

5 Auf den Fotos von berufstätigen Frauen findet sich aber noch eine andere Art des Identisch-machens, durch die die widersprüchliche Einheit von gemeinsamem Produzentenstatus und Zweitrangigkeit weiblicher Produzenten anschaulich vermittelt wird. Gemeint sind die vielen Fotos von Frauen, die hintereinander, in scheinbar ins Endlose verlängerten Reihen, die gleichen Arbeiten verrichten (am Fließband, an den Kassen der Kaufhalle zum Beispiel) - identisch in Bewegung und Körperhaltung und als einzelne austauschbar durch eine der Vielen (anderen Frauen), die die gleichen Fertigkeiten beherschen. Bei der Fotorecherche wurden keine vergleichbaren Bilder von Männem gefunden. Mănner kommen so gut wie nie in der vervielfachten Ausführung gleicher Bewegung, Handgriffe usw. ins Bild. Mănner haben besondere Aufgaben und Fähigkeiten, sie sind nicht ohne weiteres ersetz- und austauschbar, scheinen die Fotos zu sagen- auf jeden Fall machen sie anschaulich, daß weibliche Berufsarbeit »anders « ist. 
6 Diesen seit Jahren in soziologischen Untersuchungen immer emeut hervorgehobenen Fakt hat u.a. in letzter Zeit eine Erhebung zum Wert von Ehe und Familie bei Jugendlichen bestatigt. (Vgl.: Ursula Hempel und Angelika Otto, Jugendliche in der DDR au Ehe und Familie. In: Informationen des Wissenschaftlichen Rates Die Frau in der sozialistischen Gesellschafta. H.3/1988, S.6)

7 Die auf vielen Fotos feststellbare Abschwächung von Weiblichkeitsstereotypen wird im ubrigen durch Bildunterschriften, Balken und Textpassagen teil weise wieder aufgehoben Es ist, als sollte mit der massiven Beschwơrung *klassischer « Klischees und Formeln (die endlos wiederholten Kombinationen von „Charme und Logik $*$, „Schönheit und Geist, , von Sachinformation und Beschreibung des Äußeren der Frauen usw.) der Fakt verkleinert werden, daB Frauen mit dem Einstieg in die Berufstătigkeit Kenntnisse und Fähigkeiten erwerben, die sich als Sprengkrafi tradienter Ordnungen herausstellen kornten.

8 Es gibt heute in der DDR viele Frauen, die über eine hohe berufliche Qualifikation verfugen. Von den 80,3\% der berufstatigen Frauen, die Ende 1984 eine abgeschlossene Berufsausbildung hatten, waren 21.9\% Hoch- und Fachschulkader. Dennoch sind Frauen gegenwärtig noch relativ selten in innovativen wissenschaftlichen Bereichen bzw. in den Disziplinen, die den wissenschaftlich - technischen Fortschrit bestimmen, tutig. Nicht selten werden sie (häufiger als Molnner) nach abgeschlossener Ausbildung unterhalb ihrer Qualifikation eingesetzt.

9 In der NBI dagegen sind Folos von Wissenschaftlerinnen oder Leiterinnen auf mittlerer oder hoheret Ebene so selten, verglichen mit der Anzahl von Fotos ihrer männlichen Kollegen, daB die Ausnahmeposition dieser Frauen anschaulich vermittelt wird.

10. Farbfotos sind in DDR-Zeitschriften aus technischen Gründen immer noch eine Seltenheit, die Entscheidung, was farbig ins Bild kommt, sagt daher etwas uber offizielle Gewichtigkeit von Themen und Zusammenhängen aus.

11 Auf den Fotos von Frauen ist das Stereotyp der »Mutterlichkeit " fast immer vorhanden - auch die "Karrierefrauen" werden möglichst mit "mutterlichen " Zugen ins Bild gesetzt und es ist wohl kein Zufall, daB fast nur von solchen Frauen berichtet wird, die verheiratet sind und/oder Kinder haben. Bei den Männerfotos läBt sich im Laufe der Jahre ein Wechsel vom «Emährer* zum »zärtlichen Vater « feststellen, der insbesondere zum Kleinstkind eine emotionale, köpernahe Beziehung hat und es »wie eine Mutter " versorgen kann. Hier zeichnen sich moglicherweise Modifizierungen in tradierten Mämerbild ab, auf deren widerspruchliche Bedeutungen an dieser Stelle nicht eingegangen werden $k a n n$. Festgehalten werden soll hier nur, daß sich diese Modifizienungen bisher ausschließlich auf Fotos abfinden lassen, die $M$ ünner in der Familie, in der Privatsphäre zeigen: ihr Bild, das sie in offentlichen, also ihren weigentlichen « Bereichen zeigt, ist davon bisher unberühr.

12 Von den rund 5400 Fotos, die im1986er Jahrgang der beiden Zeitschriften Frauen und/oder Mynnet abbildeten, zeigten ganze 14 Frauen oder Männer bei Hausarbeiten (Kochen, Aufrăumen, Fensterputzen, Einkaufen, Abwaschen).

13 Es ist diese Einheit von Be-Deutung einer konkreten widerspruchlichen Realitat und der fortlaufenden Reproduktion eines Wahmehmungs-, Denk- und Deutungsmusters, das Hierarchie, Oberund Unterordnung, Privilegienung des Einen und Abwertung des Anderen als »naturlich« vermittelh, aus der die Virulenz des "mämlichen Blicks« erklärbar wird, auf den hin die Fotos und auch die Gesamtgestaltung der Zeitschriften ausgerichtet sind. In der FD zum Beispiel werden in der Regel die Berichte und Fotos vom aktuellen politischen Geschehen und von selbstbewuBten, kompetenten Frauen, die im Benuf erfolgreich wihren Marnes stehen bzw. als Mütter die Doppelbelastung bewaltigen weingerahmt " von einem farbigen Titelfoto, das mehr oder weniger onekkisch « eine Vertreterin des »schönen Geschlechts « zeigl, sowie - nach einer »Atempause« durch den Fortsetzungsroman - von verfuhrerisch schönen, gepflegten, dem Alltag entruckten Mannequins, die als sTraumfrauen « »geschwătzig«, wie Foucault das bezuglich der Sexualităt nannte, eine Norm propagieren: \#Weiblichkeit« als *unverüußerlicher Wert«.

14 Die im folgenden angedeuteten Zusammenhănge gehen selbstverständlich weit uber das hinaus, was das hier vorgesteltte konkrete Projekt zu leisten imstande ist; andererseits wird an ihnen sichtbar, um Veränderungen welcher Größenordnungen es geht, zu deren Bewußtwerdung dieses Projekt beitragen will. 